\title{
Compression Fractures in the Setting of Diffuse Idiopathic Skeletal Hyperostosis
}

\author{
Amila M.G Silva ${ }^{1}$, Shron Si Heng Tan ${ }^{2}$, Milindu Chanaka Makaranda ${ }^{2}$, John Li Tat Chen ${ }^{1}$ \\ ${ }^{1}$ Department of Orthopaedic Surgery, Singapore General Hospital, Singapore \\ ${ }^{2}$ Department of Orthopaedics, National University Hospital, Singapore
}

Compression fractures are the most common vertebral fractures. They involve the anterior column of the spine, and are considered stable fractures due to the presence of intact posterior ligaments that aid in resisting further collapse and deformity. They are thus often managed conservatively. We describe a series of 3 cases that were initially diagnosed as compression fractures and managed conservatively. With the abundance of compression fractures and increase in preference for conservative management of compression fractures, it is of utmost importance to recognize the possibility of other spinal co-pathologies, especially that of hyperostosis of the spine, both by clinical judgment as well as radiological analysis before embarking on conservative management, should there be under-treatment and development of complications that could have otherwise been avoided, as in the cases presented in this series.

Keywords: Compression fractures; Spinal; Diffuse idiopathic skeletal hyperostosis; Roentgenography

\section{Introduction}

Compression fractures are the most common vertebral fractures. They involve the anterior column of the spine, and are considered stable fractures due to the presence of intact posterior ligaments that aid in resisting further collapse and deformity. They are thus often managed conservatively [1]. Indeed, recent studies have shown that compression fractures can be effectively managed conservatively, with vertebroplasty and kyphoplasty offering no additional benefits to these patients. In a placebocontrolled study, patients who underwent sham surgery and vertebroplasty had similar, significant reductions in overall pain and similar improvement in function, quality of life and perceived recovery. This result is corroborated by another similar study [2-4]. These results, however, led to the under-treatment and over-diagnosis of many com- pression fractures. In this series, we present three cases of chance fractures which were misdiagnosed as compression fractures. These cases occurred within a period of two years at a single centre. We hope to emphasize the importance of excluding co-existing pathologies of the spine before labeling the fracture as a compression fracture and undertaking conservative management of these spinal trauma and injuries.

\section{Case Reports}

\section{Case 1}

A 72-year-old female, who was premorbid activities of daily living (ADL)-independent with no significant past medical history, presented to the Emergency Department on June 2011 with acute lower back pain following

Received Jul 8, 2014; Revised Oct 22, 2014; Accepted Oct 30, 2014

Corresponding author: Amila M.G Silva

Department of Orthopaedic Surgery, Singapore General Hospital, Blk 107,

Unit 08-124 Spottiswoode Park, Singapore 080107, Singapore

Tel: +65-8484-8498, Fax:+65-6224-8100, E-mail: amila157@hotmail.com 
a low energy fall on the same day. There was no radiation of pain to the lower limbs, and no neurological signs or symptoms. The pain was not aggravated or relieved by postural changes or increase in abdominal pressure. The initial roentgenogram indicated a compression fracture at T11 as shown in Fig. 1A, and the patient was managed conservatively.

The patient; however, returned to the Emergency Department 54 days after the date of injury with bilateral lower limb weakness of grade 2 across all myotomes according to the Medical Research Council (MRC) scale for muscle power. Further magnetic resonance imaging (MRI) revealed a chance fracture at T11 as shown in Fig. $1 \mathrm{~B}$ and $\mathrm{C}$, warranting surgical treatment with decompression laminectomy at T1-T12 and posterior stabilization at T8-L2. However the patient was unable to undergo the surgery as she had suffered a cerebrovascular accident. She was treated conservatively as the risk stratification was quite high. The patient was discharged 50 days after her second presentation to the emergency department. The patient then underwent rehabilitation, allowing her lower limb motor functions to improve to grade 3.

\section{Case 2}

An 85-year-old female, who was premorbid ADL-inde- pendent with no significant past medical history, presented to the Emergency Department on October 2011 following a low energy fall on the same day. There was no radiation of pain to the lower limbs, and no neurological signs or symptoms. The pain was not aggravated or relieved by postural changes or increase in abdominal pressure. As in Fig. 2A, the initial roentgenogram demonstrated a compression fracture at L3 and syndesmophytes bridging the thoracic and lumbar vertebrae. The patient was managed conservatively.

Two days after hospital admission however, the patient's bilateral lower limb motor function deteriorated to grade 0 globally according to the MRC scale for muscle power with reduced sensation across all dermatomes. Further MRI revealed a chance fracture at T11 as in Fig. 2B and C, with the patient undergoing decompression laminectomy at T1-T12 and posterior stabilization at T8-T12. The patient was then discharged 55 days after the second presentation to the emergency department, with the patient's lower limb motor functions improving to grade 3 aster postoperative rehabilitation (Fig. 2D).

\section{Case 3}

A 91-year-old male, who was premorbid ADL-independent with a background of ankylosing spondylitis (AS),
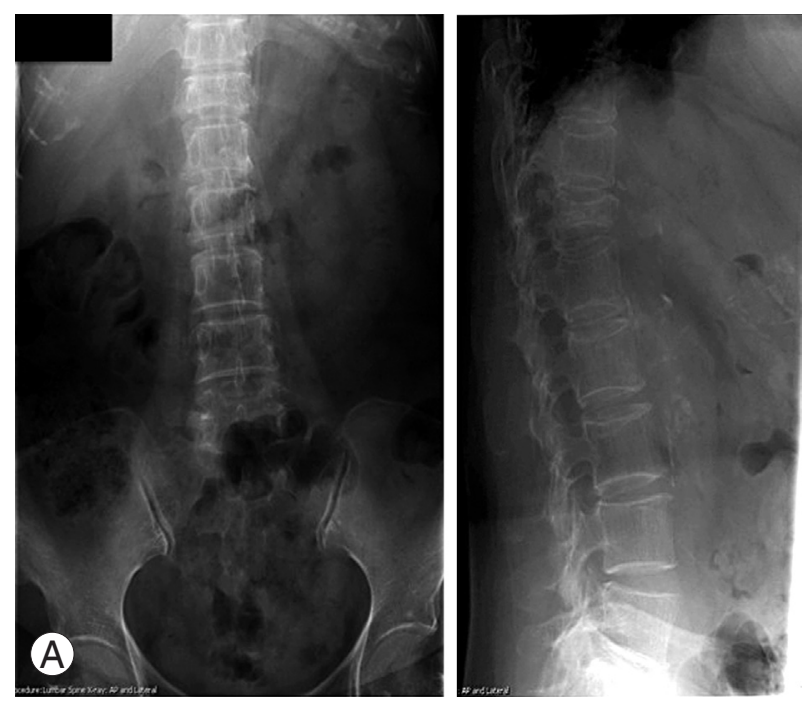

Fig. 1. Patient 1. (A) Initial roentgenogram of the patient A showing T11 chance fracture with cord compression. (B, C) Magnetic resonance imaging of patient A showing T11 chance fracture with cord compression.
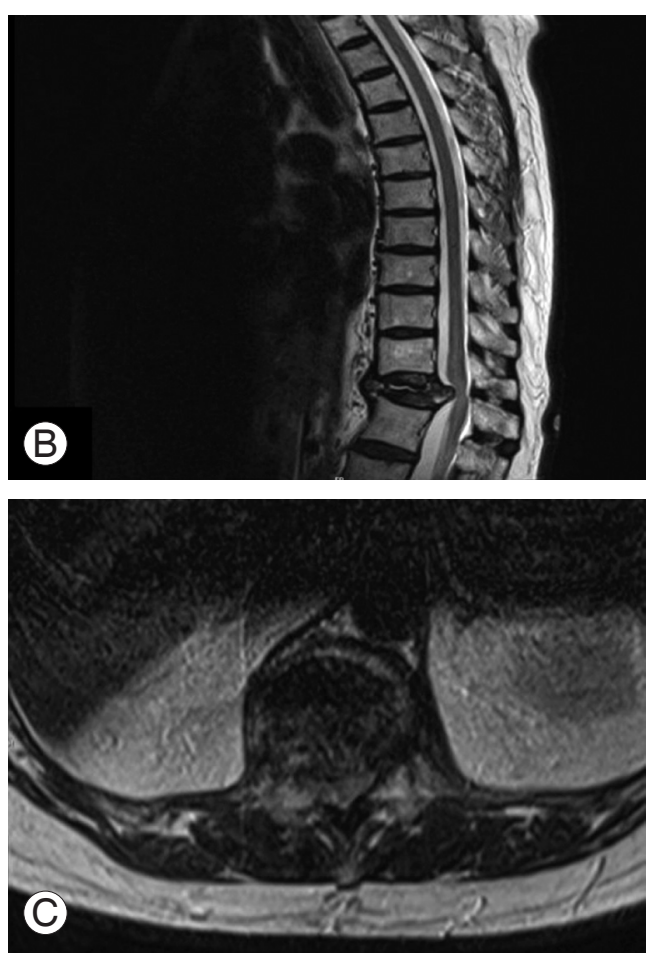


\section{ASIAN SPINE JOURNAL_ Compression fractures in the setting of diffuse idiopathic skeletal hyperostosis 631}
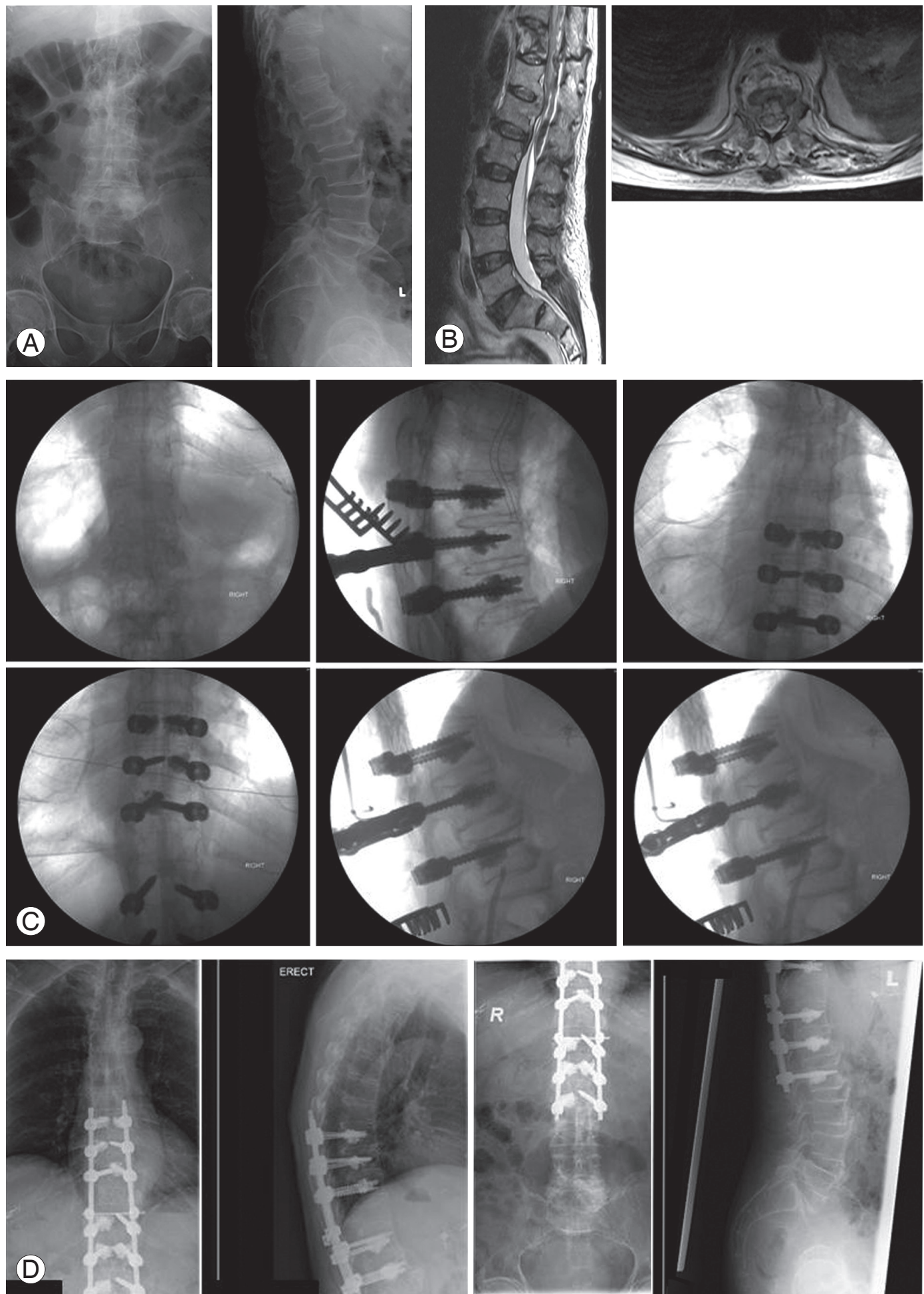

Fig. 2. Patient 2. (A) Initial roentgenogram of the patient A showing T11 chance fracture with cord compression. (B) Magnetic resonance imaging of patient A showing T11 chance fracture with cord compression (left: sagittal section, right: cross section). (C) Intraoperative image intensifier images. (D) Postoperative roentgenogram with posterior stabilization.

presented to the Emergency Department on June 2011 following a low energy fall on the same day. There was no radiation of pain to the lower limbs, and no neurological signs or symptoms. The pain was not aggravated or relieved by postural changes or increase in abdominal pressure. The initial roentgenogram demonstrated a compression 
fracture at T12-L1 as shown in Fig. 3A, and the patient was managed conservatively with bed rest and analgesia.

However, due to the persistence of severe back pain, the patient underwent MRI, revealing a chance fracture at T12-L1 as in Fig. 3B and C, with the patient undergoing posterior instrumentation and fusion 10 days after the initial presentation. The patient was then discharged 53 days after the initial presentation to the Emergency Department, with the patient able to ambulate independently at the time of discharge (Fig. 3D, E).

\section{Discussion}

Throughout history, several classifications of systems have been proposed for spinal injuries, including the frequently used Denis classification based on the threecolumn concept [5] as well as classifications according to the mechanism of injuries [6]. In the 1990s, the AO Committee for Spinal Classification reviewed these classifications and subsequently developed a more comprehensive system based on the three basic functions of a stable spine described by Whitesides [7], which reflect the ability of a stable spine to resist axial compression forces, axial distraction forces and torsional forces, as well as rotational forces around the longitudinal axis. Three basic injury types are therefore differentiated in the $\mathrm{AO}$ classification, with type A representing compression injuries, type $\mathrm{B}$ distraction injuries, and type $\mathrm{C}$ rotational injuries. Each type of injury is further subdivided into 3 groups accordingly. The severity of the injuries increases stepwise from type A to type $\mathrm{C}$ and from group 1 to group 3 , with correspondingly poorer prognosis. Specifically in this context, compression fractures are classified as type A while chance fractures are type $\mathrm{B}$ according to the $\mathrm{AO}$ classification [8].

\section{Comparison of presentations of compression and chance fractures}

By virtue of both compression fractures and chance fractures being thoracolumbar spinal fractures, patients in both clinical scenarios present with lower back pain as the main symptom, with rare neurological deficits since such fractures do not usually involve retropulsion of bone fragments into the vertebral canal $[1,2,9,10]$. However, while compression fractures occur in patients with severe osteoporosis during trivial events, in patients with moder- ate osteoporosis following minor injuries to the spine and in patients without osteoporosis in severe trauma, chance fractures are typically associated with motor vehicle accidents typically termed as 'seatbelt fractures', as well as with other mechanisms including falls, sporting events and assaults [2,9-13]. This overlapping clinical picture, coupled with the subtle radiographical features in chance fractures, could then possibly lead to misdiagnosis or delayed diagnosis of chance fractures, as in the three cases we have presented.

Radiographic evaluation of compression fractures may demonstrate the classical 'wedge' pattern of fracture. Smith and Kaufer [14] described the differentiation of chance fractures from other fractures by their disruption of the posterior elements of the spine, longitudinal separation of the disrupted posterior elements, minimal or no decrease in the anterior vertical height of the involved vertebral body, minimal or no forward displacement of the superior vertebral fragment and minimal or no lateral displacement of the fractured or superior vertebrae $[9,15]$. However, it is paramount to realize that the clinical spectrum of vertebral compression fractures and chance fractures are wide. Mild compression fractures may also have minimal wedging and decrease in anterior vertebral height while severe compression fractures with greater than $40 \%$ loss of anterior vertebral height could similarly have their posterior ligaments damaged by distraction, leading to further collapse and deformity [1]. Indeed, numerous studies have reported delayed diagnosis of chance fractures by 24 hours or more to be more than $50 \%$ of cases. Evaluation of the spine using computed tomography (CT) or MRI have thus been advocated, with recent literature recognizing CT's increased accuracy and speed in diagnosing thoracolumbar spine fractures compared to conventional radiographs, and the superiority of MRI in excluding occult injuries and spinal cord lesions before labeling the fracture as a compression fracture (Table 1) [10,14,16-21].

\section{Consequences of misdiagnosis of chance fracture as compression fractures}

Indeed, the importance of obtaining accurate diagnosis of vertebral spine fractures cannot be over-emphasized, since this diagnosis would dictate the management of choice and possibly the complications and prognosis of the fractures. Compression fractures, being stable flexion- 


\section{ASIAN SPINE JOURNAL Compression fractures in the setting of diffuse idiopathic skeletal hyperostosis 633}
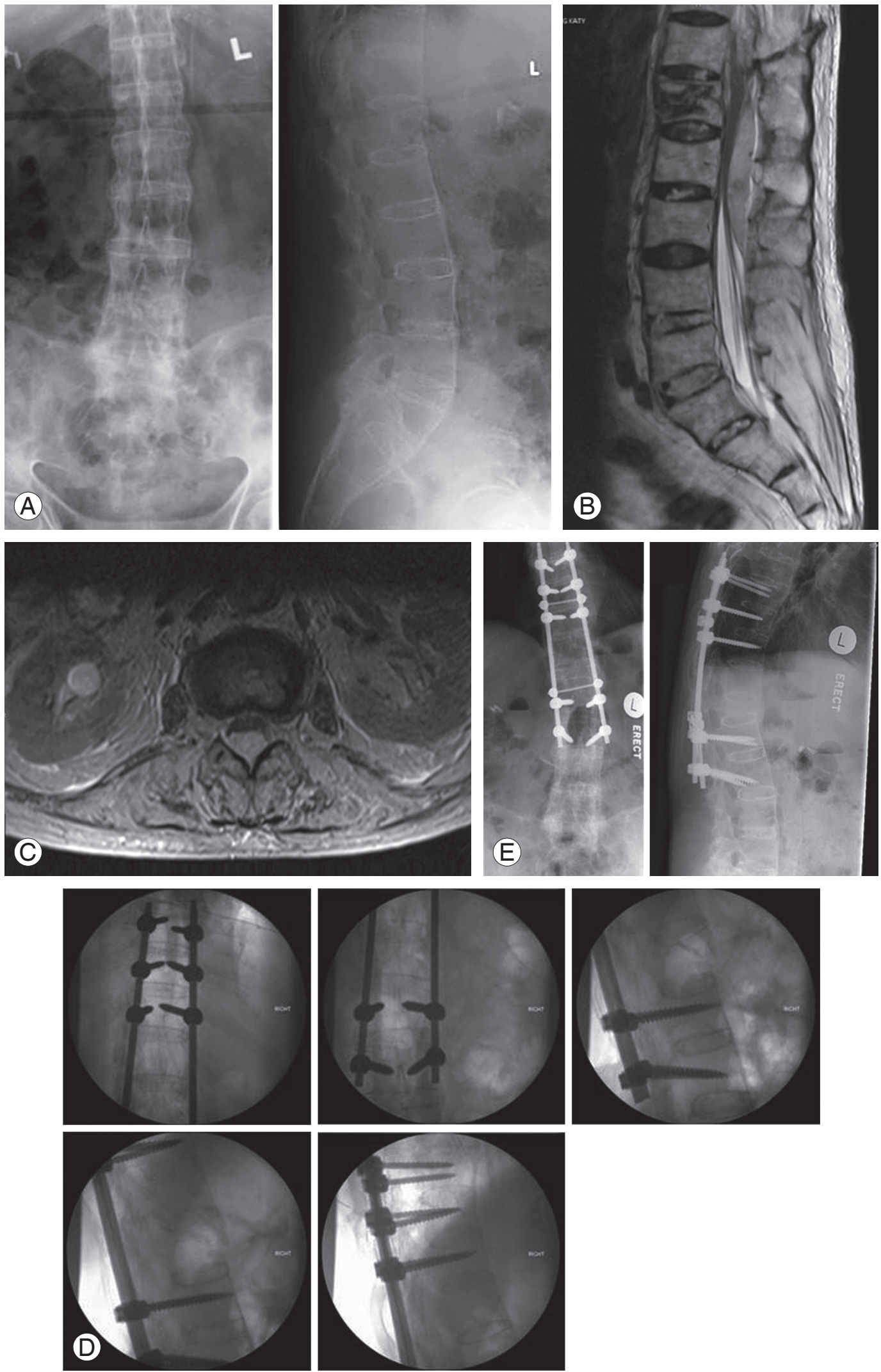

Fig. 3. Patient 3. (A) Initial roentgenogram of the third case showing $L 1$ chance fracture with cord compression. Magnetic resonance imaging of the third patient showing $\mathrm{L} 1$ chance fracture with cord compression (B) sagittal section, (C) cross section. (D) Intraoperative image intensifier images. (E) Postoperative roentgenogram with posterior stabilization. 
Table 1. The diagnostic criteria for diffuse idiopathic skeletal hyperostosis

\begin{tabular}{cl}
\hline Compulsory criteria \\
A & Preservation of disk height in the involved areas \\
B & Absence of sacroiliac erosion, sclerosis or bony fusion \\
C & Absence of bony ankylosis of the facet joints \\
Graded criteria \\
1 & Calcification or ossification along the anterolateral aspect of at least four contiguous vertebral bodies \\
\hline 2 & Calcification or ossification along the anterolateral aspect of two contiguous vertebral bodies \\
3 & Body spurs arising from the calcaneus, olecranon or patella \\
4 & Ossification of entheses \\
5 & Bony spurs palpable about the patella, elbow or heel or hard mass felt in a tendon \\
6 & $\begin{array}{l}\text { Pain and morning stiffness of the thoracic spine without substantial motion range limitation and absence of fibromyalgia tender points } \\
\text { in an adult older than } 50 \text { years of age }\end{array}$
\end{tabular}

The diagnostic criteria for diffuse idiopathic skeletal hyperostosis developed by Utsinger [21], with definite hyperostosis defined by criteria 1, probable hyperostosis defined by criteria $2+3$ to 6 , and possible hyperostosis defined by criteria $2+$ two criteria from 3 to 6 .

compression injuries, are often managed conservatively, with patients with minimal wedging treated with bed rest and analgesia, those with moderate wedging with loss of $20 \%$ to $40 \%$ of anterior vertebral height placed in a thoracolumbar brace and those with severe wedging with loss of greater than $40 \%$ of anterior vertebral height warranting possible surgical management, though controversies exist with regards to their efficacy as compared to placebo surgery. In contrast, however, chance fractures are unstable fractures produced by hyperflexion and distraction mechanisms, and would definitely require either a thoracolumbar brace to ensure no unstable deformity or posterior spinal fusion in the event of instability or neurological deficits [1]. This is especially so in the setting of a diffuse idiopathic skeletal hyperostosis (DISH) background, with multiple papers concluding that the unstable nature of even harmless appearing injuries dictates that most fractures that occur in the background of DISH be managed with fixation, as the use of orthosis is ineffective [22].

The significance of these appropriate management methods are due to the complications associated with unstable fractures, as a review by Ritchie et al. demonstrated that a delay in diagnosis and under-treatment of thoracolumbar spinal fractures contributes to neurological deficits in $10.5 \%$ of spinal fractures as compared with $1.4 \%$ when diagnosed at initial screening. This is corroborated by the three cases in our review $[10,16]$.

Indeed, fractures occuring in the setting of DISH are associated with increased instability, higher risks of complications and poorer prognosis as compared to compression fractures, thus while their clinical presentations and radiological features are similar, it is paramount to exclude other unstable fractures and co-pathologies of the spine before labeling a fracture as a compression fracture simply due to its commonality and similarity with other clinical scenarios. With the increase in preference for conservative management of compression fractures, it is of utmost importance to recognise the possibility of other spinal co-pathologies, especially that of hyperostosis of the spine, both by clinical judgment as well as radiological analysis before embarking on conservative management, should there be undertreatment and development of complications that could have otherwise been avoided, as in the cases presented in this series.

\section{Conflict of Interest}

No potential conflict of interest relevant to this article was reported.

\section{References}

1. Solomon L, Warwick D, Nayagam S. Apley's system of orthopaedics and fractures. 9th ed. London: Hodder Arnold; 2010.

2. Alexandru D, So W. Evaluation and management of vertebral compression fractures. Perm J 2012;16:46- 
51.

3. Staples MP, Kallmes DF, Comstock BA, et al. Effectiveness of vertebroplasty using individual patient data from two randomised placebo controlled trials: meta-analysis. BMJ 2011;343:d3952.

4. Miller FG, Kallmes DF, Buchbinder R. Vertebroplasty and the placebo response. Radiology 2011;259:621-5.

5. Denis F. The three column spine and its significance in the classification of acute thoracolumbar spinal injuries. Spine (Phila Pa 1976) 1983;8:817-31.

6. Gertzbein SD, Court-Brown CM. Flexion-distraction injuries of the lumbar spine: mechanisms of injury and classification. Clin Orthop Relat Res 1988;227: 52-60.

7. Whitesides TE Jr. Traumatic kyphosis of the thoracolumbar spine. Clin Orthop Relat Res 1977;(128):7892.

8. Aebi M. Classification of thoracolumbar fractures and dislocations. Eur Spine J 2010;19 Suppl 1:S2-7.

9. Kim DH, Vaccaro AR. Osteoporotic compression fractures of the spine: current options and considerations for treatment. Spine J 2006;6:479-87.

10. Bernstein MP, Mirvis SE, Shanmuganathan K. Chance-type fractures of the thoracolumbar spine: imaging analysis in 53 patients. AJR Am J Roentgenol 2006;187:859-68.

11. Birch A, Walsh R, Devita D. Unique mechanism of chance fracture in a young adult male. West J Emerg Med 2013;14:147-8.

12. Dhall SS, Tumialan LM, Mummaneni PV. Chance fracture of the second thoracic vertebra: case illustration. J Trauma 2006;60:922.

13. Davis JM, Beall DP, Lastine C, Sweet C, Wolff J, Wu D.
Chance fracture of the upper thoracic spine. AJR Am J Roentgenol 2004;183:1475-8.

14. Smith WS, Kaufer H. Patterns and mechanisms of lumbar injuries associated with lap seat belts. J Bone Joint Surg Am 1969;51:239-54.

15. Groves CJ, Cassar-Pullicino VN, Tins BJ, Tyrrell PN, McCall IW. Chance-type flexion-distraction injuries in the thoracolumbar spine: MR imaging characteristics. Radiology 2005;236:601-8.

16. Ritchie WP Jr, Ersek RA, Bunch WL, Simmons RL. Combined visceral and vertebral injuries from lap type seat belts. Surg Gynecol Obstet 1970;131:431-5.

17. Anderson S, Biros MH, Reardon RF. Delayed diagnosis of thoracolumbar fractures in multiple-trauma patients. Acad Emerg Med 1996;3:832-9.

18. Parizel PM, van der Zijden T, Gaudino S, et al. Trauma of the spine and spinal cord: imaging strategies. Eur Spine J 2010;19 Suppl 1:S8-17.

19. Hauser CJ, Visvikis G, Hinrichs C, et al. Prospective validation of computed tomographic screening of the thoracolumbar spine in trauma. J Trauma 2003;55: 228-34.

20. Keynan O, Fisher CG, Vaccaro A, et al. Radiographic measurement parameters in thoracolumbar fractures: a systematic review and consensus statement of the spine trauma study group. Spine (Phila Pa 1976) 2006;31:E156-65.

21. Utsinger PD. Diffuse idiopathic skeletal hyperostosis. Clin Rheum Dis 1985;11:325-51.

22. Bransford RJ, Koller H, Caron T. Cervical spine trauma in diffuse idiopathic skeletal hyperostosis: injury characteristics and outcome with surgical treatment. Spine (Phila Pa 1976) 2012;37:1923-32. 